Methods The total population of the city is 1,5 million population in the city of Yekaterinburg. The medical records of four city hospitals where patients with hip fractures were admitted as well as from outpatient emergency units were analysed for 1992-1997. The city administration provided statistical data on relevant population number and age distribution. Hip fracture has been defined as a fracture of the femoral neck or intertrochanteric region fracture (ICD IX code 820.0 and 820.2). There were no exclusion criteria other then the hip fracture occurring as a result of major trauma.

Results In the health care areas studied there were 2777 hip fractures: 599 males and 2178 females. The mean age of males was $69,0 \pm 11,2$ years of age and females $-76,5 \pm 9,3$ respectively $(\mathrm{p}<0,0001)$. The incidence of hip fracture for the examined period made up 132,3/100 000 population in those over 50 years $(76,9 / 100000$ for males and 164,8/100 000 for females). The ratio of male to females cases was $1: 2$. The incidence of hip fracture rose exponentially with age in both genders. The occurrence of hip fracture was maximal in subjects over 80 years old and made up 744,1/100 $000(476,9 / 100000$ males and 818,2/100 000 females). Male/female ratio was 1:1,7. The incidence of hip fracture in our population has not seasonal variation.

Conclusion More recent data showed significant differences in the incidence of hip fractures between Russian regions. The lowest rate of hip fracture was found in the western part of Russia and the highest in the Ural (Yekaterinburg). There was a large difference in incidence between regions, which suggests important environmental factors in the causation of hip fracture.

\section{OP0108 AGGRESSIVE LYMPHOMAS (DIFFUSE LARGE B CELL LYMPHOMAS) ARE OVERREPRESENTED IN RA PATIENTS}

${ }^{1} \mathrm{E}$ Baecklund, ${ }^{2} \mathrm{C}$ Sundström, ${ }^{3} \mathrm{~A}$ Ekbom, ${ }^{3} \mathrm{~N}$ Feltelius, ${ }^{3} \mathrm{~L}$ Klareskog. ${ }^{1}$ Department of Rheumatology; ${ }^{2}$ Department of Pathology, University Hospital, Uppsala; ${ }^{3}$ Department of Medicine, Karolinska Hospital, Stockholm, Sweden

\subsection{6/annrheumdis-2001.780}

Background Patients with rheumatoid arthritis (RA) have an increased risk to develop lymphomas. In a previous case-control study we found a strong association between high disease activity in RA and the risk of lymphoma.

To get a better understanding of the biological background of this connexion we examined lymphoma subtypes in RA patients. Objectives To study the distribution of lymphoma subtypes in RA patients compared to the general population.

Methods In a population-based cohort of 11,683 patients with RA in the Uppsala Health Care region in Sweden,42 cases of lymphoma were identified through record linkages with the Swedish cancer registry 1965 through 1984.

The medical records and paraffin embedded lymphoma tissues were collected.

The lymphomas were reclassified according to the recently described REAL classification. The distribution was compared to lymphoma subtypes in the general population according to the Non-Hodgkin's Lymphoma (NHL) Classification Project. ${ }^{1}$

Results One patient was excluded as the RA diagnosis was wrong. Tissues from 36 of the remaining patients were found and reviewed. NHL was found in 33 patients, Hodgkin's disease in 2 and 1 patient did not have lymphoma.

Compared with the expected there was a more than 2-fold increased number of diffuse large B-cell lymphoma (22 patients, $69 \%$ of NHL's compared with $31 \%$ expected).
Few of the patients had been treated with immunosuppressive drugs.

Conclusion The distribution of lymphoma subtypes in RA patients differs from the expected.

This supports the idea of a specific underlying mechanism behind lymphoma development in RA patients. This mechanism may also be of importance for RA development and activity. Genetics and other characteristics of those RA patients who develop lymphomas deserve further studies.

\section{REFERENCE}

1 The Non-Hodgkin's Lymphoma Classification Project: A Clinical Evaluation of the International Lymphoma Study Group. Blood 1997;89(11):3909-18

\section{OP0109 HIGHER INCIDENCE OF THROMBOEMBOLIC EVENTS AMONG PATIENTS WITH RHEUMATOID ARTHRITIS VS. OSTEOARTHRITIS, AND VS. NO ARTHRITIS, IN THE GENERAL PRACTICE RESEARCH DATABASE (GPRD)}

DJ Watson, T Rhodes. Epidemiology, Merck Research Laboratories, West Point, USA

\subsection{6/annrheumdis-2001.781}

Background Persons with Rheumatoid arthritis (RA) have been noted to have increased all-cause and cardiovascular (CV) disease mortality relative to those without RA. Data on mortality in patients with RA vs those with osteoarthritis (OA), and data on nonfatal + fatal CV incidence in patients with RA relative to those with $\mathrm{OA}$ and those with no arthritis are lacking.

Objectives To compare the incidence of all cause mortality, myocardial infarction (MI), sudden death (SD), cerebrovascular events (stroke; excluding transient ischaemic attack), CV death (fatal MI, sudden death, fatal stroke), and all thromboembolic events in RA patients compared with patients with OA but not RA, and compared with those with no arthritis, in the GPRD.

Methods The GPRD is a general practice research database that currently contains over 35 million patient years of data from the UK and represents 6\% of general practice patients in England and Wales. This was an observational cohort study of patients $>40$ years old from GPRD practices in which $>80 \%$ of patients had recorded visits. Patients with history of MI or stroke prior to beginning of follow-up were excluded. Person-time was calculated for each patient by gender, age (5 year intervals) and diagnosis (RA; OA but not RA, and no arthritis). The rate/1000 person-years was computed for each gender, age, and diagnosis stratum. For each patient, only the first incident endpoint was counted and attributed to the stratum in which it occurred. Gender and age-adjusted incidence rates and ratios of rates of each endpoint during person-time with RA vs that with OA but not RA, and vs that with no arthritis were computed using Poisson regression.

Results 594 practices contributed 6,533,474 patients to the analysis. The incidence of RA was $0.68 / 1000$ patient years in men and 1.33/1000 patient years in women. Age-adjusted rates of all endpoints were significantly increased for both men and women with RA compared to those with OA but not RA (except for sudden death, which was significantly increased in women only). Age- and gender-adjusted rate ratios (95\% CI) were: all-cause mortality 1.72 (1.67, 1.78); MI 1.32 (1.24, 1.41); SD 1.33 $(1.09,1.63)$; stroke 1.26 (1.18, 1.34); CV death 1.41 (1.24, 1.61); and all thromboembolic events 1.31 (1.25, 1.39). Similar results were seen when patients with RA were compared with those with no arthritis: age- and gender-adjusted rate ratios (95\% CI) with RA vs no arthritis were: all-cause mortality 1.60 
(1.55, 1.64); MI 1.55 (1.46, 1.65); SD 1.30 (1.07, 1.58); stroke $1.37(1.29,1.45)$; CV death $1.45(1.35,1.57)$; and all thromboembolic events 1.47 (1.41, 1.54).

Conclusion In this study patients with a diagnosis of RA were $70 \%$ more likely to die and $30-40 \%$ more likely to suffer an acute major thromboembolic event compared with patients with a diagnosis of OA but not RA. In addition, patients with a diagnosis of RA were $60 \%$ more likely to die, and 30-50\% more likely to suffer an acute major thromboembolic event compared with patients with no arthritis diagnosis.

\section{OP0110 PERFORMANCE OF A TELEPHONE QUESTIONNAIRE ADMINISTERED BY LAY PEOPLE FOR CASE DETECTION OF RHEUMATOID ARTHRITIS AND SPONDYLARTHROPATHY}

F Guillemin, A Saraux, P Fardellone, P Guggenbuhl, JM Behier, J Coste. School of Public Health EA 1124, Faculty of Medicine, Vandoeuvre-Les-Nancy, France

\subsection{6/annrheumdis-2001.782}

Background Strategies for case detection in epidemiological surveys have explored the performance of questionnaires administered by mail, self-report or in face-to-face interview by health professionals, further confirmed by a rheumatologist. The cost of such strategies and the moderate validity of these methods has led to the preliminary development of a telephone questionnaire administered by lay people that would be useful for inflammatory rheumatic diseases.

Objectives To assess the accuracy of a questionnaire for epidemiological detection of rheumatoid arthritis (RA) and spondylarthropathies (SPA) suitable for telephone survey conducted by lay people.

Methods A questionnaire was created with a list of items derived from signs, symptoms, self-report diagnosis and currently accepted epidemiological criteria for classification of RA (ACR 1987) and SPA (ESSG 1991) by a group of rheumatologists and epidemiologists. RA, SPA and control patients were recruited in rheumatology outpatient clinics in 9 university hospitals in France. All patients were examined and had diagnosis confirmed by a certified rheumatologist. In each centre, lay people from self-help group or social league were trained by a unique team of professional poll staff to conduct a telephone interview in a standard manner using this questionnaire. Each group of RA and SPA was compared to the control group for sensitivity (Se) and specificity $(\mathrm{Sp})$ of each item and overall concordance with clinical diagnosis.

Results A total of 230 RA, 175 SPA and 195 controls with mean age of 55.6, 46.4 and 55.0 years, and female/male ratio of 2.6, 0.7 and 2.2 respectively were recruited. They were interviewed on the phone by 3 to 10 people in each centre. In RA-control comparison, a set of 5 items, most belonging to the ACR criteria, were found the most informative in logistic regression analysis, with self-report diagnosis showing the highest performance $(\mathrm{Se}=0.98, \mathrm{Sp}=0.86)$. In SPA-control comparison, a few items belonging to the set of ESSG criteria were the most informative, with self-report diagnosis showing the highest performance (Se $=0.85, \mathrm{Sp}=0.97)$. The overall concordance of each set with clinical diagnosis for RA and SPA was 98\% and 93\% respectively. When self-report diagnosis was not considered, queries on peripheral joint and spinal pain contributed to detection performance most significantly, with $90 \%$ and $78 \%$ concordance with clinical diagnosis of RA and SPA respectively. When modelling the selected items in a hypothetical population with $0.5 \%$ or $1 \%$ prevalence rate, the positive predictive value ranged from 4 to $26 \%$, and the negative predictive value was $99 \%$.

Conclusion A questionnaire using clinical diagnosis self-report and presentation of epidemiological criteria in common language by lay people has been developed. Given its performances in this sample at high risk for error of specificity, it is available for case detection in epidemiological survey in the general population, provided diagnosis is further confirmed by a certified rheumatologist as part of the case ascertainment strategy.

\section{OP0123 BODY MASS INDEX AND PHYSICAL ACTIVITY AS RISK FACTORS FOR PRIMARY OSTEOARTHRITIS. A COHORT STUDY IN 50.034 PERSONS}

${ }^{1} \mathrm{~GB}$ Flugsrud, ${ }^{2} \mathrm{~B}$ Espehaug, ${ }^{2} \mathrm{LI}$ Havelin, ${ }^{1} \mathrm{~L}$ Nordsletten, ${ }^{3} \mathrm{HE}$ Meyer. ${ }^{1}$ Oslo Orthopaedic University Clinic; ${ }^{2}$ The Norwegian Arthroplasty Register, Haukeland University Hospital, Bergen, Norway; ${ }^{3}$ National Health Screening Service, Oslo

10.1136/annrheumdis-2001.783

Background As no cure exists for osteoarthritis other than operation, it is important to identify preventable aetiological factors. Objectives To describe the association between middle age body mass index (BMI) and physical activity, and later total hip replacement (THR), in a large cohort.

Methods The Norwegian Arthroplasty Register records all THRs and hip revisions performed in Norwegian hospitals. ${ }^{1}$ In three Norwegian counties the National Health Screening Service (NHSS) three times conducted a cardiovascular screening during the years 1974-88. Persons born 1925-42 were screened repeatedly at median ages 42,47 and 53 years. $^{2}$ For $89 \%$ of the participants data on body weight and height were available at the NHSS from a screening for tuberculosis, conducted during the years 1963-75 (median age 34 years). ${ }^{3}$ The registers were matched and survival analyses performed using the Cox regression model. An event was recorded when a person had his first THR for primary osteoarthritis. Follow up was 1989-98. We controlled for age at screening, body height, marital status and smoking.

Results Analysing screening data from the second cardiovascular screening only (attendance 92.8\%) we found 672 THRs. A doseresponse relationship was found between BMI and the risk for THR. Women in the highest BMI quarter had a relative risk of 3.0 (95\% CI 2.2-4.2) compared to women in the lowest quarter. The corresponding figure in men was 2.0 (95\% CI 1.5-3.0). Physical activity at work also showed a dose-response relationship. Both women and men in the most active group had a relative risk for THR of 2.1 compared to the least active group.

Analyses of BMI data from each of the four screenings indicated that the predictive value of BMI for later THR was strongest at the tuberculosis screening and decreased gradually and considerably at the following screenings.

Conclusion This study confirmed that high physical activity at work increases the risk for THR-demanding osteoarthritis in men. Contrary to earlier findings, the effect was equally strong among women in our population. We found that even for levels of BMI and physical activity at work that were well within the normal ranges an increment in these risk factors increased the risk for THR-demanding osteoarthritis. Our findings indicate that the peak impact of BMI on the development of hip osteoarthritis may be at, or before, the age of 34 years. 